monotherapy, dual-therapy with standard haemostatic techniques or rescue therapy. Haemostasis was defined as cessation of bleeding within 5 minutes of hemospray application.

Results 196 patients with UGIBs secondary to peptic ulcers were recruited (133 M, 63 F, 123/196(63\%) duodenal, 44/ $196(22 \%)$ gastric, 29/196(15\%) oesophageal). Immediate haemostasis was achieved in $171 / 196(87 \%)$ patients. The median rockall score was 7 (IQR, 6-8).

Rebleeding rates were significantly lower in forrest $2 \mathrm{a}$ relative to current predicted rebleeding rates based on forrest classifications, $2 / 21(10 \%, \mathrm{P}<0.005)$. In the $25 / 196(13 \%)$ patients who did not achieve haemostasis 18/25 (72\%) were Forrest $1 \mathrm{~b}$ ulcers. In the total cohort, 33/169(20\%) had a rebleed, median rockall score was $7(\mathrm{IQR}, 7-8)$.

Outcomes with different Forrest classifications (table 1)

\begin{tabular}{|c|c|c|c|c|}
\hline \multicolumn{5}{|c|}{ Abstract PTH-030 Table 1} \\
\hline & Forrest $1 \mathrm{a}(\mathrm{n}=37)$ & $\begin{array}{l}\text { Forrest } 1 b \\
(n=111)\end{array}$ & Forrest 2a $(n=23)$ & $\begin{array}{l}\text { Forrest } 2 b \\
(n=20)\end{array}$ \\
\hline \multirow[t]{2}{*}{ Haemostasis } & $31 / 37$ & 93/111 & $23 / 23(100 \%)$ & 19/20 \\
\hline & $(84 \%)$ & $(84 \%)$ & & $(95 \%)$ \\
\hline \multirow[t]{2}{*}{ Median Blatchford } & 13 & 12 & 12 & 12 \\
\hline & IQR: 11-14 & IQR:9-15 & IQR: 11-14 & IQR: 11-15 \\
\hline \multirow[t]{2}{*}{ Median Rockall } & 7 & 7 & 7 & 7 \\
\hline & IQR: $6-8$ & IQR:6-8 & IQR: 6-7 & IQR: $6-8$ \\
\hline \multicolumn{5}{|c|}{ Rockall score 7 predicted re-bleeding rate: $25-40 \%$} \\
\hline \multirow[t]{2}{*}{ Re-bleeding } & $8 / 31$ & $19 / 94$ & $2 / 21(10 \%)$ & $4 / 18$ \\
\hline & $(26 \%)$ & $(20 \%)$ & & $(22 \%)$ \\
\hline \multicolumn{5}{|c|}{ Rockall score 7 predicted mortality: $20-30 \%$} \\
\hline \multirow[t]{2}{*}{ 30-day mortality } & $9 / 31$ & $21 / 94$ & $3 / 21(14 \%)$ & $5 / 18$ \\
\hline & $(29 \%)$ & $(22 \%)$ & & $(28 \%)$ \\
\hline
\end{tabular}

Conclusions Hemospray is effective in achieving immediate haemostasis in peptic ulcer UGIBs. The baseline Blatchford/ rockall scores in our cohort are high with patients recruited from tertiary centres with high-risk cases. The rebleeding and mortality rates are in keeping/below the predicted rate based on the scores. The best outcome with hemospray was with forrest $2 \mathrm{a}$ ulcers.

\section{PTH-031 IS FLEXIBLE SIGMOIDOSCOPY IN THE SETTING OF NORMAL COMPUTER TOMOGRAPHIC COLONOGRAPHY NECESSARY?}

Harleen Johal*, Karen Hartery, Vikrant Kale, Vincent Cheung, Ioannis Koutsounis, Colin Ferrett, Adam Bailey. John Radcliffe Hospital, Oxford, UK

\subsection{6/gutjnl-2019-BSGAbstracts.56}

Introduction The demand to deliver rapid diagnosis of colorectal cancer (CRC) safely and effectively continues to increase. The practice of combining flexible sigmoidoscopy (FS) and CT colonography (CTC) has been driven by a concern that CTC misses some rectosigmoid cancers. NICE guidelines state that CTC can be considered as an alternative to colonoscopy for diagnosis, if a radiology service can demonstrate competency in this technique. The Oxford University Hospitals Foundation Trust OUHFT two-week wait (2WW) referral pathway for suspected CRC states that patients over 75 years, or over 60 years with chronic medical co-morbidities and symptoms suspicious of CRC should have both FS and CTC (with low dose prep for frail patients). The aim of this audit was to assess the additional cancer yield of FS in the setting of normal CTC.

Methods Analysis of all referrals $(n=941)$ meeting the above criteria over a thirteen-month period (July 2015 - August 2016) was performed. Of these referrals, 851 CTCs were reviewed and 901 FSs. Endoscopic data was obtained from reports, including findings, bowel preparation, study quality, level reached, reasons for failure and histopathology. Radiological data was obtained from CTC reports, taking into account the findings, technique, study quality and recommendations. The two data sets were then directly compared, with particular focus on the correlation between CTC and FS when identifying left-sided CRC.

Results 37 L-sided CRCs were identified on FS. The splenic flexure was only reached in $22.1 \%(n=200)$ of the studies, with poor bowel preparation being the most frequently documented reason for failure. 87 possible CRCs were identified on CTC. Of these, 34 were right-sided CRCs, 9 were leftsided suspicious polyps, and 44 were left-sided CRCs. When correlating the radiology, histopathology and endoscopic findings, there were $7 \mathrm{CRCs}$ identified on CTC that were not seen on FS. Of these, 4 were histologically proven to be CRCs that were missed due to incomplete endoscopic procedure. By comparison, only one $20 \mathrm{~mm}$ polyp, a lateral spreading tumour (LST) seen on FS was missed on CTC. 447 CTCs $(52.5 \%)$ were reported as showing benign colonic disease (e.g. diverticular disease, small polyps) and further endoscopic evaluation was advised in 27.7\% $(\mathrm{n}=124)$.

Conclusion We demonstrated that all CRCs seen on FS were identified on CTC. In the setting of normal CTC, only 1 LST was missed. By amending the OUHFT $2 \mathrm{WW}$ referral pathway, so that CTC is completed first, with subsequent endoscopic evaluation only if the CTC is inconclusive or the reporting radiologist recommends direct visualisation, we anticipate substantial resource saving without compromising diagnostic performance for colorectal cancer.

\section{PTH-032 A STUDY OF THE YIELD OF INVESTIGATION IN RECURRENT IRON DEFICIENCY ANAEMIA}

${ }^{1}$ Christopher John* ${ }^{2}$ Nicola Whiller, ${ }^{2}$ Carla Smith, ${ }^{2}$ Jonathan Snook, ${ }^{2}$ Lachlan Ayres. ${ }^{1}$ Portsmouth Hospitals Nhs Foundation Trust, UK; ${ }^{2}$ Poole Hospital NHS Foundation Trust

\subsection{6/gutjnl-2019-BSGAbstracts.57}

Introduction Recurrent iron deficiency anaemia (IDA) is a relatively common clinical condition, with potentially serious gastro-intestinal (GI) causes. Previous studies have shown that 9.6-25\% of patients with negative initial upper and lower GI investigations go on to develop recurrent IDA, with up to $55 \%$ of these patients being diagnosed with a GI pathology. ${ }^{1}$ 2 There are well established guidelines on initial investigation, but there are few data on the yield of reinvestigation for recurrent IDA. The aim of this study was to determine the diagnostic yield from investigating recurrent IDA, using a larger dataset than the few previous smaller studies.

Methods The electronic patient records of the prospectively maintained IDA database at Poole Hospital NHS Foundation Trust were retrospectively examined. Patients were categorised as recurrent IDA if they re-presented a year or more after their initial investigation. Data were collected on index and 
repeat endoscopic and/or radiological investigations and any supplementary small bowel investigations.

Results 693 patients with IDA were included from January 2016 to September 2017. 86 patients had recurrent IDA. Of those with recurrent IDA, a cause was found in 20 patients (23\%), including 4 malignancies (4.6\% of all recurrent IDA), 3 cases of Crohn's disease, along with vascular lesions and Upper GI ulceration. For all patients with recurrent IDA and those in whom a cause was identified mean age was 67.6 years and 59.9 years respectively; the mean interval between presentations was 5.5 years (range: 1.1-15.1) and 5.7 years (range: 1.8-13) respectively (NS). There was no significant difference between the haemoglobin levels of patients with a cause found (97 g/L) compared patient where no cause was found $(99.1 \mathrm{~g} / \mathrm{L})$. In those with recurrent IDA 11/86 (12.8\%) of patients had causative pathology identified on repeat routine upper and lower GI investigation, 9/86 (10.5\%) had a cause identified following small bowel investigation. The yield of small bowel investigation was $30 \%$ in recurrent IDA

Conclusions A significant proportion of patients have GI pathology on re-investigation of recurrent IDA, including malignancy in $4.6 \%$. We suggest recurrent IDA is re-investigated with bi-directional endoscopy initially, with consideration of small bowel investigation.

\section{REFERENCES}

1.. Soon A, Cohen BL, Groessl EJ, et al. Long-Term Outcomes and Prognostic Factors for Patients with Endoscopy-Negative Iron Deficiency Dig Dis Sci 2013;58:488.

2. McLoughlin MT, Tham TC. Long-term follow-up of patients with iron deficiency anaemia after a negative gastrointestinal evaluation. Eur J Gastroenterol Hepatol 2009;21:872-876.

\section{PTH-033 FCSEMS FOR LIVER TRANSPLANT BILIARY STRICTURES ARE ASSOCIATED WITH A HIGH RISK OF POST-ERCP PANCREATITIS}

${ }^{1,2}$ Chris Johnston*, 'Nicholas Church, 'Samantha Bow, ${ }^{1,2}$ Stephen Wigmore. ${ }^{1}$ Royal Infirmary of Edinburgh, Edinburgh, UK: ${ }^{2}$ University of Edinburgh, Edinburgh, UK

\subsection{6/gutjnl-2019-BSGAbstracts.58}

Introduction Benign biliary strictures are a common complication of liver transplantation, mainly as a result of constriction at the site of biliary anastomosis or ischaemic cholangiopathy. Placement of fully covered self-expanding metal stents (FCSEMS) by ERCP has been shown to be an effective treatment for benign and malignant biliary strictures and offers advantages over plastic stents of greater patency rates and the potential for stricture remodeling. The aim of this study was to assess the incidence of post-ERCP pancreatitis following placement of FCSEMS for benign biliary strictures after liver transplantation in a high-volume centre.

Methods Retrospective analysis of prospectively maintained local databases was performed. Endoscopy reports were reviewed for every ERCP (any indication) performed for liver transplant recipients between 1stJanuary 2014 and 1st January 2018. Patient outcomes were gathered from electronic patient records. Severity of pancreatitis was graded according to the Revised Atlanta Classification. Statistical comparison of two groups was performed with Fisher's exact test.

Results Over a four-year period, 36 out of 393 consecutive liver transplant recipients underwent ERCP for treatment of benign biliary strictures. A total of 97 ERCPs were performed for this patient group (mean 2.7 per patient, range 1-13). Placement of temporary fully covered self-expanding metal stents successfully achieved long-term stricture resolution in $92 \%$ of patients (22/24). However, ERCP involving placement of a first FCSEMS was associated with a considerably higher rate of post-ERCP pancreatitis than any other ERCP performed in this study population $(34.5 \%$ vs $2.8 \%, \mathrm{P}<0.0001)$; $89 \%(8 / 9)$ of episodes of pancreatitis were classified as mild, $11 \%(1 / 9)$ as severe.

Conclusions In the experience of a single high-volume centre, placement of FCSEMS by ERCP is an effective treatment for the management of benign biliary strictures, but one that is associated with a particularly high risk of pancreatitis. Patients should give informed consent accordingly. Further research into the mechanisms behind this effect (e. g. sudden occlusion of a normal pancreatic duct) and the effectiveness of additional specific prophylactic measures is underway.

\section{PTH-034 ENDOSCOPIC APOLLO OVERSTITCH SYSTEM IN MANAGEMENT OF ESOPHAGEAL LEAKS; THE FIRST REPORTED UK CASE SERIES}

Ahmed Ghanem, Vasha Kaur*, Krishna Moorthy, Marta Mano Lopes, Dav Bansi. St. Mary's Hosptal, London, UK

\subsection{6/gutjnl-2019-BSGAbstracts.59}

Introduction Esophageal leaks, either after anastomosis following esophagectomy in malignancy or after esophageal perforation (Boerhaave syndrome), have a mortality rate of $35 \%$ which can rise to $100 \%$ if not diagnosed early. Traditional management involves adequate resuscitation, broad spectrum antibitics, parenteral nutrition, percutaneous drainage and timely surgical intervention. The Apollo Overstitch is a minimally invasive endoscopic suturing device which can be mounted over a twin-channel gastroscope and allows full thickness suturing without the need for surgery. It is utilised in bariatric endoscopy but is also very effective in closing perforations and fistulas as well stent fixation to reduce the risk of stent migration. Here we describe the first case series in the United Kingdom of management of esophageal leaks by the Apollo OverStitch device.

Methods Of the 3 cases of esophageal leaks, one case was from an anastomosis after esophagectomy in malignancy and the other 2 were esophageal perforation (Boerhaave syndrome). In addition to the clinical diagnosis of a critically ill patient, esophageal leaks and perforation were diagnosed by contrast enhanced CT scan. Initial management was conservative (antibiotics, antifungals and TPN) due to high respiratory requirements. Gastroscopy under screening was undertaken to identify the leak position. Biological glue was used to close the leak in one case. In another the perforation was sutured using the Apollo device. In all 3 cases a Taewong Beta stent was inserted across the leak/perforation which was then fixed proximally with placement of further sutures to successfully prevent stent migration.

Results Patient recovery was uneventful in all 3 cases, with early introduction of oral intake. In an average of eight weeks post-operatively, patients underwent a repeat gastroscopy and stent removal. Direct visualisation and intra-procedure contrast testing demonstrated an intact oesophagus with no further leak and this was confirmed by contrast enhanced CT. We have also just successfully treated a fourth patient with stent insertion and fixation. 